\title{
Statt Schrittmacher nur noch Pillen?
}

\author{
Die Häufigkeit des plötzlichen Herztods bei Patien- \\ ten mit Herzinsuffizienz hat sich in den letzten $\mathbf{2 0}$ \\ Jahren fast halbiert - auch ohne Herzschrittmacher.
}

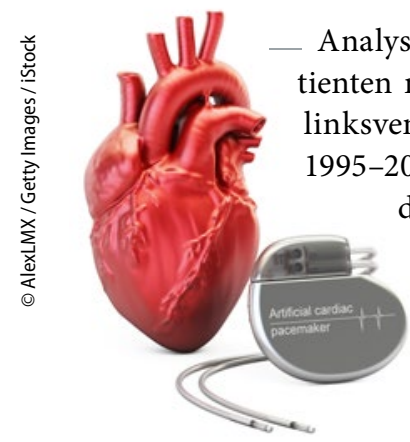

Vielen schwachen Herzen hilft ein ICD.

\section{Hier steht eine Anzeige.}

\author{
Springer
}

Herzinsuffizienz und eine schlechtere Herzfunktion.

In der Gesamtschau verringerte sich das Auftreten des plötzlichen Herztods in 20 Jahren um 44\% ( $\mathrm{p}=0,03)$. In den frühen Studien um 1995 lag die Häufigkeit in den ersten 90 Tagen bei $2,4 \%$, in den späteren bis 2014 nur noch bei 1,0\%. Die Zeit, die seit der Diagnose der Herzinsuffizienz bereits vergangen war, spielte keine Rolle für die Ereignisrate.

- Shen L et al. Declining risk of sudden death in heart failure. New Engl J Med. 2017;377:41-51

\section{KOMMENTAR}

Die kardiologischen Fachgesellschaften empfehlen einen ICD derzeit für Patienten mit Herzinsuffizienz und linksventrikulärer Auswurffraktion von $\leq 35 \%$. Man bedenke aber, dass der hier dokumentierte dramatische Rückgang des plötzlichen Herztods in einem Patientenkollektiv auftrat, aus dem Patienten mit bereits implantiertem Schrittmacher ausgeschlossen waren. Die Zahl der Patienten, die im weiteren Verlauf ein solches Gerät erhielten, ist nicht bekannt, aber wohl gering.

Deshalb folgern die Autoren, dass der bemerkenswerte Rückgang des plötzlichen Herztods bei herzinsuffizienten Patienten nicht den ICD zu verdanken ist, sondern der konsequenten konservativen Behandlung mit ACE-Hemmern, $A T_{1}$-Rezeptorblockern, Betablockern und Mineralokortikoidantagonisten. So kommen sie zu der provokanten, aber nachvollziehbaren These, dass es heute schwierig sein dürfte, noch eine Patientengruppe zu definieren, in der man einen therapeutischen Nutzen der ICD-Implantation erwarten könne. Sie postulieren, dass zumindest die in den Leitlinien geforderte konservative Therapie von drei Monaten für das Remodeling des Herzens verlängert und die ICD-Implantation zurückgestellt werden sollte. 